Department of Infectious Diseases and Infection Control, Skane University Hospital, Kioskgatan 17, 22185, Lund, Sweden

Correspondence to: U Karlsson ulf.karlsson@med.lu.se Cite this as: BMJ \$ \{year\};371:m3944 http://dx.doi.org/10.1136/bmj.m3944 Published:

\section{Covid-19: risks to healthcare workers and their families}

\author{
Mistakes made in the first wave must not be repeated in the second \\ Ulf Karlsson, Carl-Johan Fraenkel
}

Since the beginning of the coronavirus 2019 (covid-19) pandemic, healthcare workers have shown a remarkable resilience and professional dedication despite a fear of becoming infected and infecting others. ${ }^{1}$ In a linked paper (doi:10.1136/bmj.m3582), Shah and colleagues now report robust and concerning findings regarding the risks of covid-19 among health workers and their households. ${ }^{2}$

In a large register based cohort study, comprising the entire Scottish healthcare workforce, the authors compared the risk of covid-19 related hospital admission between patient facing and non-patient facing workers, their household members, and the general population. Absolute risks were low, but during the first three months of the pandemic patient facing healthcare workers were three times more likely to be admitted with covid-19 than non-patient facing healthcare workers. Risk was doubled among household members of front facing workers, in analyses adjusted for sex, age, ethnicity, socioeconomic status, and comorbidity.

Previous work reported similar risks for covid-19 among healthcare workers, ${ }^{3} 4$ but the new study provides the most comprehensive estimate to date of the risk of more serious disease, and it is the first to report risk to household members. The reasons for the observed increase in risk-likely multifactorial-need to be explored to help to guide safety improvements in healthcare settings.

During lockdowns, most essential workers are unable to protect themselves by working from home. Furthermore, insufficient physical distancing is a leading contributor to any work related covid-19 outbreak. ${ }^{5}$ Consequently, workers in sectors such as transport and social care are also at increased risk of covid-19, although healthcare workers have been shown repeatedly to be at highest risk. ${ }^{5}$

During the first wave of the pandemic, overstretched healthcare systems left health workers in hard hit countries struggling with long working hours, fatigue, and extreme psychological stress. Rapidly vanishing supplies, national lockdowns, and a feeding frenzy on the open market for personal protective equipment (PPE) led to shortages. ${ }^{7}$ Healthcare workers often had to care for patients with suspected or confirmed covid-19 infection without proper training or adequate PPE. This contributed to an increased risk to healthcare workers during the initial phase of the pandemic. 389

Superspreading events, a hallmark of previous coronavirus outbreaks, contribute substantially to community transmission of covid-19 and to work related clusters. ${ }^{5}$ Most countries struggled with insufficient testing capacity in the first months of the pandemic, which hampered early detection of emerging outbreaks and implementation of infection control measures. Increasing experience now suggests that every suspected healthcare associated infection should trigger a bundle of immediate infection control measures, including extensive screening for severe acute respiratory syndrome coronavirus 2 (SARS-CoV-2), quarantining of all patients on the affected ward, physical distancing between personnel, and use of reinforced PPE during all contact with patients on the affected ward, in order to prevent larger outbreaks. ${ }^{10-12}$

Most, but not all, studies report increased risks for health workers caring for patients with covid-19. 31314 Working in intensive care units is not associated with an increased risk of infection, possibly owing to the protection afforded by high level PPE or to the decrease in infectivity that occurs in the later stages of the illness, even among critically ill patients. ${ }^{13} 15$ The greatest risk to healthcare workers may be their own colleagues or patients in the early stages of unsuspected infections when viral loads are high. ${ }^{12}$

Most studies to date, including Shah and colleagues' study, have evaluated risks to healthcare workers during the early phases of the pandemic. Advances since then may have reduced the risks, although further confirmatory studies are needed. Such advances include greater knowledge of transmission dynamics and the impact of asymptomatic and pre-symptomatic infections, ${ }^{16}$ better access to effective PPE, improved testing capabilities, optimised triage systems, implementation of new infection control measures such as continuous mask use in hospitals, ${ }^{17}{ }^{18}$ and faster outbreak alerts and responses.

High quality prospective studies evaluating new prevention and control practices will be important to guide improvements in our approach to protecting healthcare workers and their families, ${ }^{19}$ including those from ethnic minority communities who have the highest risks of infection and poor outcomes, widening workplace inequality. ${ }^{3}$ The international community must support efforts by the World Health Organization to secure adequate supplies of PPE and covid-19 tests for low and middle income countries. An effective vaccine, if and when available, must be distributed fairly and healthcare workers must be prioritised globally. In accordance with United Nations Sustainable Development Goals, we must ensure the protection and security of all health workers in all settings. ${ }^{20}$ Competing interests: The BMJ has judged that there are no disqualifying financial
ties to commercial companies. The authors declare the following other interests: none.

Provenance and peer review: Commissioned; not peer reviewed 
1 Liu Q, Luo D, Haase JE, etal. The experiences of health-care providers during the COVID-19 crisis in China: a qualitative study. Lancet Glob Health 2020;8:e790-8.

doi: 10.1016/S2214-109X(20)30204-7 pmid: 32573443

2 Shah ASV, Wood R, Gribben C, etal. Risk of hospital admission with coronavirus disease 2019 in healthcare workers and their households: nationwide linkage cohort study. BMJ 2020;371:m3582.

3 Nguyen LH, Drew DA, Graham MS, etalCOronavirus Pandemic Epidemiology Consortium. Risk of COVID-19 among front-line health-care workers and the general community: a prospective cohort study. Lancet Public Health 2020;5:e475-83. doi: 10.1016/S2468-2667(20)30164-X pmid: 32745512

4 Chou R, Dana T, Buckley DI, Selph S, Fu R, Totten AM. Epidemiology of and Risk Factors for Coronavirus Infection in Health Care Workers: A Living Rapid Review. Ann Intern Med 2020;173:120-36. doi: 10.7326/M20-1632 pmid: 32369541

5 European Centre for Disease Prevention and Control. COVID-19 clusters and outbreaks in occupational settings in the EU/EEA and the UK. 2020. https://www.ecdc.europa.eu/en/publications-data/covid-19-clusters-and-outbreaks-occupational-settings-eueea-and-uk.

6 Mutambudzi M, Niedzwiedz CL, Macdonald EB, etal. Occupation and risk of severe COVID-19: prospective cohort study of 120075 UK Biobank participants.2020. https://www.medrxiv.org/content/10.1101/2020.05.22.20109892v2.

7 Burki T. Global shortage of personal protective equipment. Lancet Infect Dis 2020;20:785-6 doi: 10.1016/S1473-3099(20)30501-6 pmid: 32592673

8 Kua J, Patel R, Nurmi E, etal. HealthcareCOVID: A national cross-sectional observational study identifying risk factors for developingsuspected or confirmed COVID-19 in UK healthcare workers.2020. https://www.medrxiv.org/content/10.1101/2020.08.28.20182295v1.

9 Zhang Z, Liu S, Xiang M, etal. Protecting healthcare personnel from 2019-nCoV infection risks: lessons and suggestions. Front Med 2020;14:229-31. doi: 10.1007/s11684-020-0765-x pmid: 32212058

10 Huff HV, Singh A. Asymptomatic transmission during the COVID-19 pandemic and implications for public health strategies. Clin Infect Dis 2020;ciaa654. doi: 10.1093/cid/ciaa654 pmid: 32463076

11 Rickman HM, Rampling T, Shaw K, etal. Nosocomial transmission of COVID-19: a retrospective study of 66 hospital-acquired cases in a London teaching hospital. Clin Infect Dis 2020;ciaa816 doi: 10.1093/cid/ciaa816 pmid: 32562422

12 Lucey M, Macori G, Mullane N, etal. Whole-genome sequencing to track SARS-CoV-2 transmission in nosocomial outbreaks. Clin Infect Dis 2020;ciaa1433. doi: 10.1093/cid/ciaa1433 pmid: 32954414

13 Eyre DW, Lumley SF, O’Donnell D, etalOxford University Hospitals Staff Testing Group. Differential occupational risks to healthcare workers from SARS-CoV-2 observed during a prospective observational study. Elife 2020;9:e60675. doi: 10.7554/eLife.60675 pmid: 32820721

14 Hunter E, Price DA, Murphy E, etal. First experience of COVID-19 screening of health-care workers in England. Lancet 2020;395:e77-8. doi: 10.1016/S0140-6736(20)30970-3 pmid: 32333843

15 van Kampen JJA, van de Vijver DAMC, Fraaij PLA, etal. Shedding of infectious virus in hospitalized patients with coronavirus disease-2019 (COVID-19): duration and key determinants.2020. https://www.medrxiv.org/content/10.1101/2020.06.08.20125310v1.

16 Cevik M, Marcus JL, Buckee C, Smith TC. SARS-CoV-2 transmission dynamics should inform policy. Clin Infect Dis 2020;ciaa1442. doi: 10.1093/cid/ciaa1442 pmid: 32964919

17 World Health Organization. Advice on the use of masks in the context of COVID-19: interim guidence, 6 April 2020. 2020. https://apps.who.int/iris/handle/10665/331693.

18 Seidelman JL, Lewis SS, Advani SD, etal. Universal masking is an effective strategy to flatten the severe acute respiratory coronavirus virus 2 (SARS-CoV-2) healthcare worker epidemiologic curve. Infect Control Hosp Epidemiol 2020;1-2. doi: 10.1017/ice.2020.313 pmid: 32576336

19 World Health Organization. Protocol for assessment of potential risk factors for 2019-novel coronavirus (COVID-19) infection among health care workers in a health care setting. 2020. https://www. who.int/publications/i/item/protocol-for-assessment-of-potential-risk-factors-for2019-novel-coronavirus-(2019-ncov)-infection-among-health-care-workers-in-a-health-caresetting.

20 World Health Organization. Working for health and growth - investing in the health workforce. 2016. https://www.who.int/hrh/com-heeg/reports/en/.

This article is made freely available for use in accordance with BMJ's website terms and conditions for the duration of the covid-19 pandemic or until otherwise determined by BMJ. You may use, download and print the article for any lawful, non-commercial purpose (including text and data mining) provided that all copyright notices and trade marks are retained. 